\title{
Explorando o uso de regras de correspondência condicional na busca evolutiva por autômatos celulares classificadores de densidade binária
}

\author{
A. L. L. Cardoso ${ }^{1}$ ， P. P. Balbi $i^{1,2}$ \\ ${ }^{1}$ Programa de Pós-Graduação em Engenharia Elétrica e Computação \& \\ ${ }^{2}$ Faculdade de Computação e Informática \\ Universidade Presbiteriana Mackenzie \\ Rua da Consolação 896, Consolação - 01302-907 São Paulo, SP \\ cardosoonline@hotmail.com, pedrob@mackenzie.br
}

\begin{abstract}
Cellular automata are discrete systems, fundamentally based upon local interactions, which, even though simple, may yield universal computation. A classical problem to probe the computational capacity of CAs is the density classification task, whose objective is to decide the prevailing bit in an arbitrary binary sequence. Here we investigated the efficacy that a recently proposed representation of $C A$ rules would have in that task, since the new structure of the search space, induced by this new representation, might prove beneficial. Evolutionary searches were performed in different formulations of the density task, even in larger dimensionalities of the space, led to limited impact on the efficacy of the rules found. The results contrast with those found in the literature, pointing at limitations of the representation scheme employed.
\end{abstract}

Resumo. Autômatos celulares (ACs) são sistemas discretos, fundamentados em interações locais que, mesmo simples, podem ser capazes de computabilidade universal. Um problema clássico de estudo da capacidade computacional dos ACs é a tarefa de classificação da densidade, na qual se objetiva determinar o bit predominante em uma sequência binária arbitrária. Investigou-se aqui a eficácia que uma representação de regras de ACs recentemente proposta na literatura poderia ter nessa tarefa, já que a nova estrutura do espaço de busca, induzida pela nova representação, poderia ser benéfica. Buscas evolutivas realizadas em diferentes formulações do problema, inclusive em maiores dimensionalidades do espaço, evidenciaram impacto restrito na eficácia das regras encontradas. Tal resultado contrasta com os disponíveis na literatura, apontando limitações do esquema de representação utilizado.

\section{Introdução}

Autômatos celulares (ACs) são sistemas que a partir de operações locais simples podem manifestar comportamentos globais complexos. São sistemas discretos, no qual cada unidade de processamento (chamada célula) age localmente somente com sua vizinhança, mas em conjunto, podem produzir esses comportamentos complexos, inclusive manifestando computabilidade universal. Muitas aplicações de ACs foram efetuadas em domínios distintos tais como: vida artificial [Sipper 1994], nano-computação [Peper et al. 2009] e simulações de moléculas [Sahu et al. 2012]. 
Um problema amplamente estudado em ACs é o da Tarefa de Classificação de Densidade (TCD), em inglês Density Classification Task . O seu objetivo é encontrar uma regra de $\mathrm{AC}$ unidimensional que diante de uma configuração inicial binária aleatória, classifique corretamente se essa configuração possui maior quantidade de células com estado 1 . Se a maioria for 1 , o autômato deve evoluir a uma configuração homogênea onde todas as células devem possuir o estado 1 e permanecer assim para demais evoluções; ao contrário, analogamente, evoluir a 0 . Uma tarefa simples para um algoritmo em sistema com processamento centralizado, mas não é trivial para ACs devido às suas interações estritamente locais. O presente trabalho é sobre como o uso de uma nova reapresentação de ACs pode ser aplicada na TCD. Denominada de Regras de Correspondência Condicional (RCC), em inglês Conditionally Matching Rules, demonstrou sucesso em diversos experimentos em diferentes espaços de ACs com vários estados [Bidlo 2016]. Em um desses experimentos, explorou-se o problema de replicação de ciclos em ACs de duas dimensões e obteve-se a melhor velocidade de replicação até o momento. Avaliou-se também o incremento de dimensionalidades (mais estados), aumentando de 4 para 8 estados, pois mais informação por célula poderia encontrar mais cenários de replicação. Essa mudança de estrutura do espaço é atrativa, pois novos caminhos poderiam levar às regras pretendidas. Esse novo esquema de representação admite redundâncias, ou seja, diferentes representações podem apontar para uma mesma regra de AC.

Para a TCD essa nova representação pode ser útil, mesmo considerando que a dimensionalidade do espaço aumente devido ao acréscimo de parâmetros dela advindo, como se verá adiante. De fato, no presente trabalho aumentamos a dimensionalidade do espaço de busca ainda mais, através do uso de estados auxiliares, os quais, diferentemente dos binários, não fazem parte do problema que está sendo resolvido, tendo apenas o papel de permitirem a criação de novos caminhos no espaço de busca, sendo, portanto, eliminados ao final da evolução temporal do AC. Existem diversos trabalhos onde se avaliam como novas representações de AC são aplicadas a TCD: Fukś [Fukś and Procyk 2020] explorou formas generalizadas da TCD utilizando 3 estados no intuito de encontrar, analogamente, regras para essas generalizações. Fatès [Fatès 2015] também explorou o uso de estados auxiliares viabilizando uma solução simples para a tarefa de sincronização, que é um problema também muito estudado e possui algumas características semelhantes ao da TCD. A diferença entre os dois é que em vez de se chegar a um comportamento fixo, o comportamento resultante oscila ciclicamente entre reticulados homogêneos entre os dois estados binários. O presente trabalho está organizado da seguinte forma: a Seção 2 trata do referencial teórico com noções de AC, RCCs e TCD. A Seção 3 descreve a metodologia e como foram realizados os experimentos com as suas justificativas. Na Seção 4 temos os resultados e discussão dos experimentos. Finalmente, na Seção 5 apresenta a conclusão e sugestões de trabalho futuros.

\section{Referencial Teórico}

\subsection{Autômatos Celulares}

Autômatos Celulares (ACs) são sistemas dinâmicos que a partir de suas operações locais, podem fazer emergir comportamentos globais e complexos [Wolfram 2002]. Sua ação é totalmente discreta, no tempo, no espaço, variáveis de estado e cada uma de suas unidades básicas de processamento (as células) se definem num reticulado regular, e interagem apenas com outras na suas vizinhanças. 
Os ACs podem ser definidos por uma 4-tupla $A=(S, N, f, d)$, no qual $S$ representa o conjunto de estados $S=\{0,1, \ldots, k-1\} ; N=\left(a_{1}, a_{2}, \ldots, a_{n}\right)$ representa a vizinhança, com $N \in \mathbb{Z}^{d} ; f: S^{n} \rightarrow S$ é a sua função ou regra local de transição de estados; e $d$ a sua dimensão. Um reticulado $C$ é composto por células nos quais seus estados estão definidos por $S$ onde $C \in \mathbb{Z}^{d}$ e $d>0$. O raio $(r)$ de um $\mathrm{AC}$ refere-se à abrangência da vizinhança. No caso unidimensional, o tamanho da vizinhança é dado por $w=2 r+1$. A função de transição $f$ recebe como parâmetro a vizinhança, que inclui a célula central, e especifica qual será o novo estado da célula central. Uma regra de AC se define a partir de todas as possíveis configurações de vizinhança. A regra é aplicada em todas as células do reticulado, de forma síncrona, gerando uma evolução temporal no reticulado, sendo que em $t=0$ temos a configuração inicial (CI). A condição de contorno rege como as extremidades do reticulado se comportam; no presente trabalho considera-se a condição periódica, em que a primeira e última células do reticulado são consideradas conectadas, implicando que todas as configurações são cíclicas.

Todas as regras podem ser representadas de forma $k$-ária, ou pelo número decimal correspondente. Por exemplo, a regra 30 (do chamado espaço elementar) pode ser representada de forma binária por $00011110_{2}$, já que sua tabela de transição de estados é: $111 \rightarrow 0,110 \rightarrow 0,101 \rightarrow 0,100 \rightarrow 1,011 \rightarrow 1,010 \rightarrow 1,001 \rightarrow 1,000 \rightarrow 0$. Qualquer espaço de regras de ACs é composto por $k^{k^{2 r+1}}$ regras o que evidencia que o custo para ser examinado cresce exponencialmente, o que dificulta a busca de alguma regra com comportamento especifico ou com uma dada propriedade. Os ACs aqui pesquisados possuem $r=3, k=\{2,3,4,8\}$ e $d=1$.

\subsection{Regras de Correspondência Condicionais}

Uma nova forma de representação de regras de ACs proposta principalmente em [Bidlo 2016] demonstrou seu potencial quando aplicada a diferentes problemas de ACs. Uma RCC é composta de duas partes: 1) uma parte condicional e 2) um estado de retorno. A parte condicional corresponde diretamente à quantidade de células da vizinhança. Cada parte condicional é um par ordenado composto por um operador condicional, que pode ser $=, \neq, \leq$, ou $\geq$, e um estado de avaliação. A Figura 1 ilustra graficamente como essa composição é feita.

Uma função de transição, ou regra, de AC possui um conjunto de combinações de vizinhanças que denotam as suas regras transições. Comparativamente, uma função de transição baseada em RCCs, possui uma determinada quantidade de RCCs. Note que uma função de transição baseada em RCCs pode ser transformada no formato convencional tabular (onde $=$ seria o operador condicional para todas RCCs), o que facilitaria uma análise comparativa entre as duas representações.

$\mathrm{Na}$ avaliação da vizinhança, cada célula é comparada à sua respectiva parte condicional da RCC seguindo o processo: se todas as partes condicionais da RCC resultarem verdadeiro, o próximo estado é o estado de retorno; caso contrário, ou seja, nenhuma RCC da função de transição baseada em RCCs corresponder à vizinhança em questão, então a célula permanecerá com o mesmo estado para a próxima evolução temporal. Para ilustrar, em um AC de $r=1$, temos uma função de transição com 3 RCCs sendo: RCC $1,(\neq 1, \neq 1, \leq 0) \rightarrow 1$; RCC $2,(=0, \neq 0, \leq 1) \rightarrow 0$; e RCC $3,(\neq 0, \geq 0, \leq 1) \rightarrow 1$, como mostrado na Figura 1. Neste exemplo, usamos condição de contorno fixa, ou seja, 


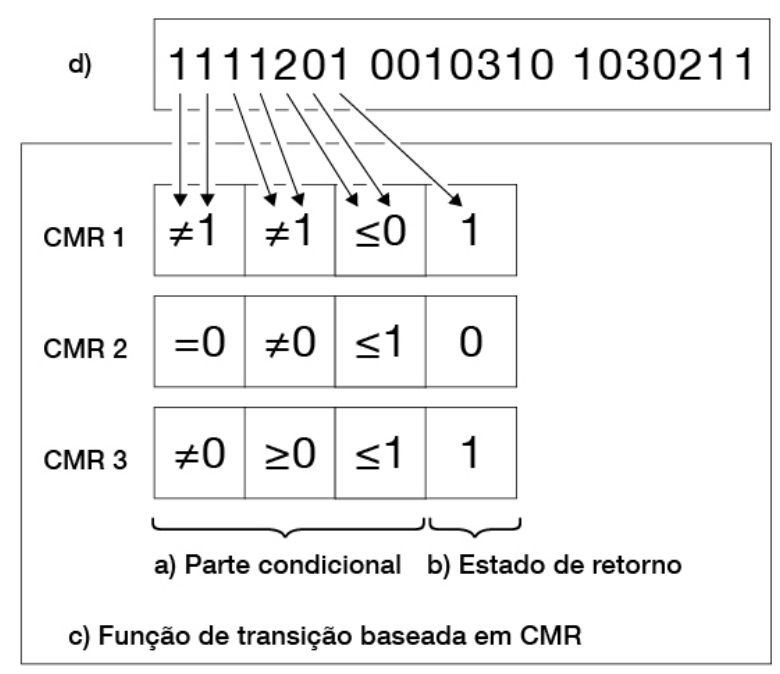

Figure 1. Uma RCC é composta por uma parte condicional (a) e um estado resultado (b). Essa função de transição baseada em RCCs (c) é composta por 3 RCCs. A representação da função de transição por números inteiros pode ser vista em (d).

fixam-se 0s nas extremidades do reticulado. Caso a CI seja 1101, e considerando-se que $a_{x}$ é o estado $a$ da célula na posição $x$ no reticulado, o estado da primeira célula seria $a_{1}$ e a do contorno fixo à sua esquerda $a_{0}$, então a vizinhança de $a_{1}$ é $\left(a_{0}, a_{1}, a_{2}\right)$ que será $(0,1,1)$. Avaliando a RCC 1 , a condição $a_{0} \neq 1$ é verdadeira pois $0 \neq 1$, já $a_{1} \neq 1$ é falsa pois $1 \neq 1$. Logo a RCC 1 não é validada com a vizinhança e não há a necessidade de avaliar as demais partes condicionais. Seguindo avaliando a RCC 2, as condições $a_{0}=0$, $a_{1} \neq 0$ e $a_{2} \leq 1$ são verdadeiras, o que causa essa regra a validar com a vizinhança, logo o resultado se dá pelo estado de retorno da RCC 2, no caso, 0 . Como a RCC 2 foi validada, não há necessidade de avaliação da RCC 3 terminando a avaliação para aquela célula.

\subsection{Tarefa de Classificação da Densidade}

A Tarefa de Classificação da Densidade, também conhecida como problema da maioria, tem um objetivo simples: encontrar uma regra de AC binário de uma dimensão que determine se uma CI arbitrária cíclica contem uma maioria de quantidade de 1s. Sejam um reticulado $C=\left(a_{1}, a_{2}, \ldots, a_{n}\right)$, com $n$ ímpar, $S=\{0,1\}$ e $\rho_{1}$ a densidade de 1 s em $C$; então, se $\rho_{1}>0.5$ depois de uma certa quantidade de iterações, todos os elementos em $C$ devem convergir para uma configuração de 1s; caso contrário, $0 \mathrm{~s}$. Mesmo o alfabeto sendo binário, é possível formular o problema utilizando-se um AC com maior quantidade de estados, que cumpririam um papel de estados auxiliares, apenas participando temporariamente da evolução do AC; aqui também realizamos experimentos nesse contexto. Uma extensa revisão sobre a TCD é disponível em [de Oliveira 2014].

A necessidade de processamento global da TCD não é trivial para um $\mathrm{AC}$, uma vez que este se baseia estritamente em interações locais. Portanto, como as células 1s estão distribuídas por todo o reticulado, a regra deve produzir alguma forma de cooperação. Land e Belew [Land and Belew 1995] provaram que a TCD não pode ser resolvida perfeitamente com qualquer $\mathrm{AC}$ de dois estados. Todavia, ainda está em aberto qual seria a regra que atingiria a melhor eficácia e esse desafio ainda é muito estudado em dias atuais. O teste com raio $r=3$ e reticulado $C=149$ virou uma re- 
ferência para experimentos da TCD. Várias regras foram encontradas que desempenham na TCD, como a de Gacs, Kurdyomuv e Levin (projetada manualmente), a de Mitchell, Hraber e Crutchfield (obtida por algoritmos genéticos) entre outras, como a regra de Julié e Pollack (obtida por um processo coevolutivo), que permaneceu por 10 anos como a regra de melhor eficácia, até que finalmente, Wolz e de Oliveira [Wolz and de Oliveira 2008] encontraram (por meio de um processo coevolutivo sofisticado), a regra 337607298446901146542393000444934784552 (WdO1) com $\approx 88.99 \%$ de classificações corretas, e que permanece até hoje como a provável melhor regra conhecida.

\section{Metodologia}

Um Algoritmo Genético (AG) simples foi utilizado como método de busca. O indivíduo é representado por uma sequência de RCCs e criados aleatoriamente no início do AG. A aptidão do indivíduo é dada pela quantidade de classificações corretas de CIs na TCD. As CIs são criadas ao começo de cada geração com uma distribuição de Bernoulli, o que gera uma amostra centrada em torno de $\rho=0.5$. As RCCs são codificadas como números inteiros idênticos aos exemplo da Figura 1. A quantidade de RCCs é de 20 e será explicada na Seção 4. Os experimentos possuem $r=3$ e reticulado de comprimento $R=35$, em vez de $R=149$ devido a recursos computacionais. Seguindo os experimentos na TCD descritos em [Wolz and de Oliveira 2008] são necessárias $2.5 R$ evoluções temporais para convergir, então fixamos uma quantidade de 100 iterações para o AC.

Executou-se uma avaliação de robustez que expõe um indivíduo a cenários mais intensos de avaliação, como diferentes formas de distribuição de densidade de $1 \mathrm{~s}$, maior quantidade de CIs e maiores configurações. Duas condições de parada na evolução do AC foram aplicadas que reduziram consideravelmente o tempo de término dos experimentos: uma interrompe configurações fixas e a outra periódicas. Experimentos iniciais foram com 8 indivíduos, seguindo o trabalho descrito em [Bidlo 2016], mas nenhuma melhora de eficácia média foi observada. Incrementalmente, aumentou-se a quantidade de indivíduos até 50, quando se obteve o melhor resultado. Iniciamos com 100 gerações, mas não desempenharam para experimentos com muitos estados, de forma que depois de 20 experimentos sem nenhuma evolução de eficácia média detectada, passou-se a 500 gerações onde se obteve incremento na eficácia média. Elitismo de 1 foi utilizado para preservar o melhor indivíduo durante as gerações. O método de seleção foi o de torneio de tamanho 2, em vez de 4 (conforme usado em [Bidlo 2016]), para diminuir a pressão seletiva. Não foi utilizado crossover. A mutação era aplicada da seguinte forma: expandia-se as RCCs em um vetor e um número aleatório entre 0 e 4 era escolhido. $\mathrm{O}$ resultado era a quantidade de números inteiros que seriam alterados no indivíduo, respeitando o formato válido da RCC. Se o número 0 fosse selecionado, o indivíduo não sofreria mutação, sendo clonado. Comparava-se então o indivíduo original com sua versão mutada e este somente seria adicionado na nova geração caso sua aptidão fosse maior que o original. A evolução do AG ocorria até um máximo de gerações pré-definido.

Para avaliar o comportamento dos estados auxiliares utilizados, eles foram monitorados de duas formas, sem distingui-los entre si. Primeiramente foi monitorada a quantidade de estados auxiliares no último passo de tempo da evolução do $\mathrm{AC}$ o que não demonstrou relação com a eficácia. Posteriormente, eles foram monitorados durante a sua completa evolução do AC e calculadas a porcentagem estados auxiliares existentes ao 

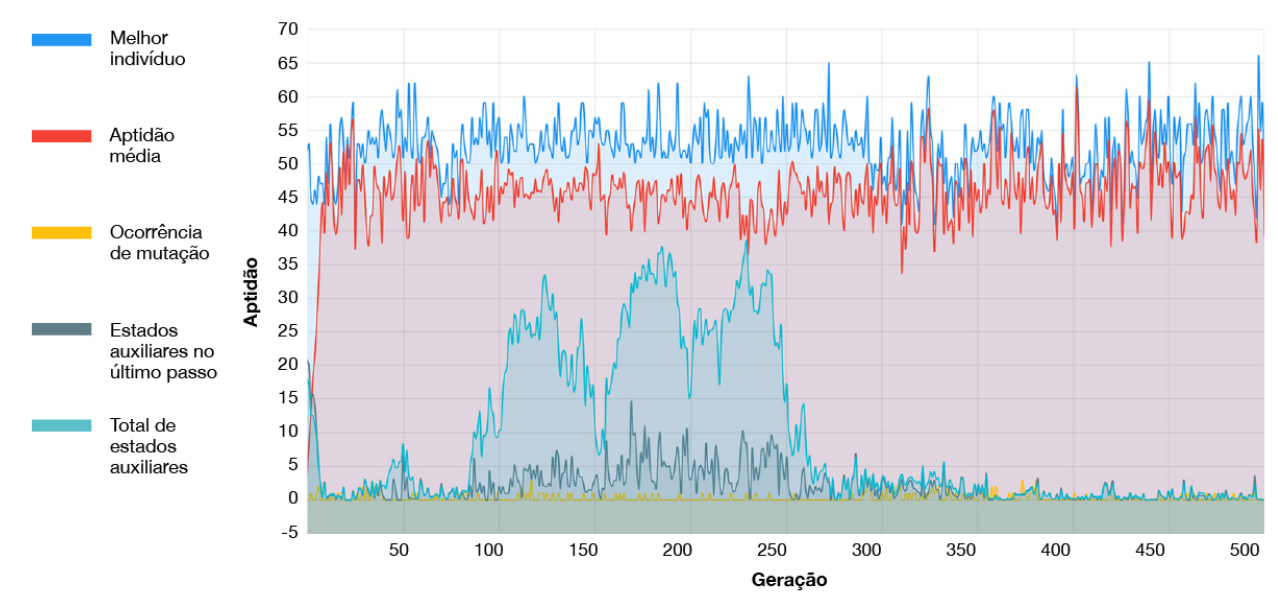

Figure 2. Impacto dos estados auxiliares na busca evolutiva. Entre a geração $\mathbf{7 0}$ e 270, um visível aumento do total de estados auxiliares é observado, mas sem impacto na aptidão média.

final.

\section{Resultados}

Um total de 40 experimentos foram realizados usando-se diferentes quantidades de estados. Testes com 2, 3, 4 e 8 estados com 10 experimentos foram feitos. Duas métricas de eficácia foram obtidas: a aptidão média dos indivíduos da população, e a elite dos $5 \%$ melhores indivíduos, aqui referida simplesmente por elite. A Tabela 2 explicita os resultados obtidos.

Para saber a quantidade de regras RCCs dentro de uma função de transição, executamos 15 experimentos com uma quantidade de 15, 20 e 25 RCCs por função de transição. Com menos que 10 RCCs a maioria das evoluções entrava em um comportamento fixo ou periódico sem resolver a TCD e, com mais de 30 RCCs, tornavam-se muito custosas computacionalmente. O melhor resultado foi com 20 RCCs.

$\mathrm{Na}$ avaliação de robustez selecionava-se o indivíduo de melhor aptidão no final da geração de um dos experimentos, o qual era então submetido a diferentes tamanhos de configurações: 51, 149, 255 e 999. Avaliamos duas distribuições: a distribuição de Bernoulli e a quase-balanceada, na qual $\rho_{1} \approx 0.5$. A eficácia não decaiu proporcionalmente ao tamanho do reticulado. Já a diferença entre as duas distribuições de densidade era esperada pois sabe-se que a quase-balanceada representa a pior condição de acerto na TCD [de Oliveira 2014]. A queda de eficácia nas duas distribuições ocorre com relativa proporção, o que pode ser observado na Tabela 1.

Os experimentos iniciais de 4 estados foram considerados sem resultados aparentes em termos de eficácia média. Um exemplo pode ser visto na Figura 2, onde tem-se um aumento significativo na participação dos estados auxiliares, sem, no entanto, alteração na aptidão média ou melhor indivíduo. Em todos os experimentos, uma rápida estabilização da aptidão média chegava a 50\%. Esse comportamento ocorre por volta das primeiras 30 gerações. O motivo é que nas gerações iniciais rapidamente os melhores indivíduos dominam a população. 


\begin{tabular}{c|r|r|ccc|}
\hline \multicolumn{1}{c}{$C$} & \multicolumn{3}{c}{$\begin{array}{c}\text { Distribuição } \\
\text { Uniforme (\%) }\end{array}$} & $\begin{array}{c}\text { Distribuição } \\
\text { Quase-Balanceada (\%) }\end{array}$ \\
\hline \multirow{3}{*}{100000} & 51 & 100 & 85.88 & 61.77 \\
& 149 & 425 & 81.48 & 54.97 \\
& 255 & 750 & 79.75 & 53.44 \\
& 999 & 3000 & 75.86 & 51.29 \\
\hline
\end{tabular}

Table 1. Análise de robustez de um indivíduo evoluído com RCCs.

Para referência, os testes binários foram executados. A média de aptidão não produziu bons resultados (55.27\%), mas a média da elite $(81.59 \%)$ foi razoável. O conjunto de experimentos de 3 estados, apresentou a melhor aptidão média (57.54\%). Esta foi maior que a de 2 estados. A aptidão da elite $(78.09 \%)$ foi menor que a do experimento binário. Os testes com 4 estados foram os primeiros testes a serem executados e foram utilizados para definir a quantidade de RCCs. Uma pequena diminuição da aptidão média $(57,11 \%)$ ocorreu e também uma queda da aptidão da elite $(75.65 \%)$. Finalmente, os experimentos de 8 estados foram feitos e levaram ao pior resultado, com aptidão média de $(49.12 \%)$ e também da elite $(59.33 \%)$. Embora se necessitasse uma rotina de testes mais extensa, criam-se indícios que o aumento da dimensionalidade possui limitações na busca evolutiva para a TCD usando RCCs. Foi mostrado que a técnica de RCCs pode ser aplicada também ao problema da TCD, levando a resultados compatíveis com os de outras regras apresentadas na literatura.

\begin{tabular}{|c|c|c|c|c|c|c|c|c|c|c|c|c|c|}
\hline Estado & Tipo & 1 & 2 & 3 & 4 & 5 & 6 & 7 & 8 & 9 & 10 & Média & $\begin{array}{l}\text { Desvio } \\
\text { Padrão }\end{array}$ \\
\hline \multirow{2}{*}{2} & Média & \begin{tabular}{|l|l|}
61.77 \\
\end{tabular} & \begin{tabular}{|l|l|}
53.90 \\
\end{tabular} & \begin{tabular}{|l|l|}
55.13 \\
\end{tabular} & 52.84 & \begin{tabular}{|l|l|}
46.90 \\
\end{tabular} & \begin{tabular}{|l|l|}
53.95 \\
\end{tabular} & \begin{tabular}{|l|l|}
55.22 \\
\end{tabular} & 59.14 & \begin{tabular}{|l|l|}
58.53 \\
\end{tabular} & 55.32 & 55.27 & 3.84 \\
\hline & Elite & 89.50 & 77.90 & 78.20 & 90.70 & 72.90 & 77.50 & \begin{tabular}{|l|}
77.60 \\
\end{tabular} & 80.00 & 83.40 & 88.20 & 81.59 & 5.73 \\
\hline \multirow[b]{2}{*}{3} & Média & \begin{tabular}{|l|}
68.17 \\
\end{tabular} & 54.35 & \begin{tabular}{|l|}
53.79 \\
\end{tabular} & 66.73 & \begin{tabular}{|l|}
56.00 \\
\end{tabular} & 52.50 & \begin{tabular}{|l|}
55.10 \\
\end{tabular} & 64.52 & 54.00 & 52.20 & 57.54 & 5.87 \\
\hline & Elite & \begin{tabular}{|l|}
90.70 \\
\end{tabular} & 78.70 & $\mid 77.30$ & 88.80 & \begin{tabular}{|l|}
74.80 \\
\end{tabular} & 77.90 & \begin{tabular}{|l|}
79.70 \\
\end{tabular} & 87.50 & 77.40 & 48.10 & \begin{tabular}{|l|l|}
78.09 \\
\end{tabular} & 11.30 \\
\hline & Média & \begin{tabular}{|l|}
54.42 \\
\end{tabular} & \begin{tabular}{|l|}
44.66 \\
\end{tabular} & \begin{tabular}{|l|}
53.53 \\
\end{tabular} & 70.72 & \begin{tabular}{|l|}
70.27 \\
\end{tabular} & \begin{tabular}{|l|}
46.98 \\
\end{tabular} & \begin{tabular}{|l|}
58.22 \\
\end{tabular} & 45.26 & 56.02 & 71.05 & 57.11 & 9.86 \\
\hline & Elite & 71.80 & 71.90 & 84.10 & 89.20 & 89.30 & \begin{tabular}{|l|}
52.90 \\
\end{tabular} & \begin{tabular}{|l|}
79.30 \\
\end{tabular} & 49.20 & 79.20 & 89.60 & 75.65 & 13.81 \\
\hline & Média & 44.88 & 54.42 & 54.25 & \begin{tabular}{|l|}
59.67 \\
\end{tabular} & \begin{tabular}{|l|}
46.62 \\
\end{tabular} & \begin{tabular}{|l|l|}
44.84 \\
\end{tabular} & \begin{tabular}{|l|}
45.32 \\
\end{tabular} & 50.63 & 45.26 & 45.30 & 49.12 & 5.05 \\
\hline & Elite & $\mid 54.70$ & 75.30 & 71.20 & 88.00 & 56.60 & 45.62 & $\mid 46.11$ & 61.95 & 48.23 & 45.55 & 59.33 & 13.89 \\
\hline
\end{tabular}

Table 2. Experimentos realizados.

\section{Conclusão}

O presente trabalho avaliou autômatos celulares representados por Regras de Correspondência Condicional, com o objetivo de encontrar ACs com bom desempenho na Tarefa de Classificação de Densidade, certamente o problema de decisão mais estudado na literatura no contexto da análise da capacidade computacional dos ACs. Diferentes formulações do problema em distintas dimensionalidades foram analisadas utilizando essa representação.

Os experimentos mostraram que, mesmo encontrando indivíduos com desempenho significativo, a representação possui limitações ainda não evidenciadas na literatura. As execuções demonstraram que o aumento de dimensões reduz a eficácia média do melhor indivíduo encontrado ao final da evolução.

Embora o emprego de uma rotina de testes mais exaustiva, como o emprego de maior quantidade de gerações na busca evolutiva, pudesse levar os resultados obtidos a 
alguma melhora, nada indica que se pudesse obter ganho expressivo. De fato, os experimentos realizados criam indícios de que a representação possa ter limitações intrínsecas para a TCD, e, por extensão, talvez a problemas correlatos, como o problema da paridade, em que se deseja determinar a paridade da quantidade de 1s na configuração inicial do AC. No entanto, mesmo com esse comportamento, alguns indivíduos foram encontrados com eficácia compatível com algumas abordagens reportadas na literatura, conforme relatadas em [de Oliveira 2014]. Além disso, nota-se que, como a representação com RCCs é mais compacta que a convencionalmente utilizada, baseada nas transições de estado diretamente, a representação com RCCs admite claro ganho de memória.

O mais importante que se vislumbra à frente seria se tentar mapear que classes de problemas com ACs seriam mais naturalmente adequados para a representação com RCCs. O que nosso estudo sugere, em sintonia com os resultados da literatura, é que, talvez essa representação se mostre tão mais eficaz quanto maior o número de estados definidos no problema.

\section{Agradecimentos}

Este trabalho se insere nos projetos de pesquisa da CAPES STIC-AmSud (CoDANet) no. 88881.197456/2018-01 e Mackenzie-PrInt no. 88887.310281/2018-00, e no projeto CNPq PQ 305199/2019-6.

\section{References}

Bidlo, M. (2016). On routine evolution of complex cellular automata. IEEE Transactions on Evolutionary Computation, 20(5):742-754.

de Oliveira, P. P. (2014). On density determination with cellular automata: Results, constructions and directions. Journal of Cellular Automata, 9(5-6):357-385.

Fatès, N. (2015). Remarks on the cellular automaton global synchronisation problem. In International Workshop on Cellular Automata and Discrete Complex Systems, pages 113-126. Springer.

Fukś, H. and Procyk, R. (2020). Explorations of ternary cellular automata and ternary density classification problems. arXiv preprint arXiv:2002.08924.

Land, M. and Belew, R. (1995). No perfect two-state cellular automata for density classification exists. Physical review letters, 74:5148-5150.

Peper, F., Lee, J., Adachi, S., and Isokawa, T. (2009). Cellular nanocomputers: a focused review. International Journal of Nanotechnology and Molecular Computation (IJNMC), 1(1):33-49.

Sahu, S., Oono, H., Ghosh, S., Bandyopadhyay, A., Fujita, D., Peper, F., Isokawa, T., and Pati, R. (2012). On cellular automata rules of molecular arrays. Natural Computing, 11(2):311-321.

Sipper, M. (1994). Studying artificial life using a simple, general cellular model. Artificial Life, 2(1):1-35.

Wolfram, S. (2002). A new kind of science, volume 5. Wolfram media Champaign, IL.

Wolz, D. and de Oliveira, P. P. B. (2008). Very effective evolutionary techniques for searching cellular automata rule spaces. J. Cellular Automata, 3:289-312. 